\title{
The microbial content of raw and pasteurized cow milk as determined by molecular approaches
}

\author{
Lisa Quigley, ${ }^{*} \dagger^{1}$ Robert McCarthy, ${ }^{*} \dagger^{1}$ Orla O'Sullivan, ${ }^{*}$ Tom P. Beresford, $\dagger$ Gerald F. Fitzgerald, $† \ddagger$ \\ R. Paul Ross, ${ }^{*} \ddagger$ Catherine Stanton, ${ }^{*} \ddagger$ and Paul D. Cotter ${ }^{*}{ }^{2}$ \\ *Teagasc Food Research Centre, Moorepark, Fermoy, Cork, Ireland \\ †Microbiology Department, University College Cork, Cork, Ireland \\ $\ddagger$ Alimentary Pharmabiotic Centre, Cork, Ireland
}

\begin{abstract}
The microbial composition of raw and pasteurized milk is assessed on a daily basis. However, many such tests are culture-dependent, and, thus, bacteria that are present at subdominant levels, or that cannot be easily grown in the laboratory, may be overlooked. To address this potential bias, we have used several culture-independent techniques, including flow cytometry, real-time quantitative PCR, and high-throughput DNA sequencing, to assess the microbial population of milk from a selection of commercial milk producers, pre- and postpasteurization. The combination of techniques employed reveals the presence of a previously unrecognized and diverse bacterial population in unpasteurized cow milk. Most notably, the use of high-throughput DNA sequencing resulted in several bacterial genera being identified in milk samples for the first time. These included Bacteroides, Faecalibacterium, Prevotella, and Catenibacterium. Our culture-independent analyses also indicate that the bacterial population of pasteurized milk is more diverse than previously appreciated, and that nonthermoduric bacteria within these populations are likely to be in a damaged, nonculturable form. It is thus apparent that the application of state-of-theart approaches can provide a detailed insight into the bacterial composition of milk and could potentially be employed in the future to investigate the factors that influence the composition of these populations.
\end{abstract}

Key words: high-throughput DNA sequencing, flow cytometry, quantitative PCR

\section{INTRODUCTION}

Milk harbors a complex microbial community, including microorganisms of industrial importance, that possess health-promoting features and that are of con-

Received February 12, 2013.

Accepted April 22, 2013.

${ }^{1}$ These authors contributed equally to this work.

${ }^{2}$ Corresponding author: paul.cotter@teagasc.ie cern from a food quality or safety perspective. Thus, the milk microbiota is the focus of constant attention. Such testing occurs daily on both raw and pasteurized milk and is governed by a variety of methods and standards across different jurisdictions. The microbial composition of milk is influenced by several different parameters such as, in the case of raw milk, the microorganisms present in the teat canal, on the surface of teat skin, in the surrounding air, in feed, as well as other environmental factors including housing conditions, the quality of the water supply, and equipment hygiene (Verdier-Metz et al., 2009, 2012; Vacheyrou et al., 2011; Braem et al., 2012). The microbiota of pasteurized milk is thought to be determined by the percentage of thermoduric bacteria that survive pasteurization temperatures and by the bacteria associated with postpasteurization contamination, which include psychrotrophic bacteria, such as Pseudomonas (Ternström et al., 1993; Fromm and Boor, 2004). The techniques used to identify and count the bacterial populations present generally involve culturing on agar media and are labor intensive and time consuming. Furthermore, microorganisms that cannot be easily cultured in the laboratory, or are present as subdominant populations, are not detected using these approaches (Paszyn'ska-Wesołowska and Bartoszcze, 2009). Indeed, comparative culture-based and culture-independent (flow cytometry) analysis of identical milk samples have provided significantly different results (Gunasekera et al., 2002). Other culture-independent techniques, and in particular those which are DNA-based, provide a means of examining the bacterial composition of milk without introducing culture-based biases. These DNAbased approaches have included denaturing gradient gel electrophoresis and single stranded conformation polymorphisms. These allow a comparison of the relative diversity of different bacterial populations and can, to some extent, reveal the identity of specific components (Callon et al., 2007; He et al., 2009). Methods, such as quantitative real-time PCR (qPCR), have increasingly been employed, which permit rapid identification and quantification, albeit only of specific target microbes 
(Rodríguez-Lázaro et al., 2005; He et al., 2009). Even more recently, significant developments have occurred in the field of microbial ecology as a consequence of the development of culture-independent analysis via high-throughput DNA sequencing. This approach can provide a more in-depth insight into the diversity and dynamics of entire microbial communities (Sogin et al., 2006; Andersson et al., 2008; Quigley et al., 2011; Delmont et al., 2012). In a few exceptional cases this technology has been applied to dairy-based environments, such as cheese (Masoud et al., 2011; Alegría et al., 2012; Quigley et al., 2012b) and kefir (Dobson et al., 2011). The aim of this study was to provide a detailed insight into the microbial composition of raw and pasteurized milk, sourced from a variety of facilities using high-throughput DNA sequencing in combination with other culture-independent approaches.

\section{MATERIALS AND METHODS}

\section{Strains and Culture Conditions}

The strains used in this study were Lactococcus lactis $\mathrm{HP}$ and Pseudomonas aeruginosa PAO-1 (Teagasc Food Research Centre Culture Collection). Lactococcus lactis was grown in M17 broth with glucose (GM17) at $30^{\circ} \mathrm{C}$ and $P$. aeruginosa was grown in Luria broth $(\mathbf{L B})$ and on $1 \% \mathrm{LB}$ agar plates at $37^{\circ} \mathrm{C}$.

\section{Flow Cytometry}

The viability of microbial populations found in raw and pasteurized milk samples obtained from the Teagasc Research Centre, Moorepark, dairy herd were investigated using flow cytometry. Flow cytometry analyses were performed using a FACSCanto II flow cytometer (BD Biosciences, San Jose, CA) using 2 air-cooled lasers, a $20-\mathrm{mW}$ solid state laser (emission, $488 \mathrm{~nm}$ ) and a $17 \mathrm{~mW}$ HeNe laser (emission, $633 \mathrm{~nm}$ ), and 5 sensors for the detection of forward and sideward light scatter: green $(525 \mathrm{~nm})$, yellow $(575 \mathrm{~nm})$, and far red $(695 \mathrm{~nm})$ fluorescence. Flow cytometry detector and threshold settings were established using a series of control studies. Here, raw milk (from the Moorepark dairy herd) spiked with L. lactis were subjected to heat treatments at $80^{\circ} \mathrm{C}$ for $30 \mathrm{~min}$ and $3 \mathrm{~h}$. Prior to analysis, proteins and lipids were removed from milk samples using a modified version of the procedure described by (Gunasekera et al., 2000). Briefly, milk was treated with 20 arbitrary units/mL of proteinase K (SigmaAldrich Co., Arklow, Ireland), and $500 \mu \mathrm{L}$ of $0.05 \%$ Triton X-100 (Sigma-Aldrich Co.) at $37^{\circ} \mathrm{C}$ with shaking for $45 \mathrm{~min}$. Samples were centrifuged at $17,000 \times g$ at room temperature for $10 \mathrm{~min}$, following which the milk fat layer and supernatant were removed. The resulting pellet was washed twice and resuspended in $1 \mathrm{~mL}$ of filtered $(0.22 \mu \mathrm{m})$ PBS (Invitrogen, Life Technologies, Carlsbad, CA). Viability testing was performed using BD Cell Viability Kit (BD Biosciences). Samples were stained with propidium iodide $(41 \mathrm{n} M)$ for $10 \mathrm{~min}$ on ice, followed by thiazole orange staining $(8.5 \mu M)$ for $15 \mathrm{~min}$ in the dark. Cell samples were delivered at the low flow rate, corresponding to 500 to 1,000 cells/s, until 10,000 cells were measured. Fluorescence signals were recorded by using the detector settings forward scatter $(\mathrm{FSC})=300$; side scatter $(\mathrm{SSC})=300$; fluorescein isothiocyanate $($ FITC) $=600$; and propidium iodide $=500$. A threshold was set at an SSC signal of 300 to reduce background noise deriving from cellular debris and traces of milk components remaining following treatment. Following the generation of threshold parameters, commercial raw and pasteurized milk samples were assayed to assess the relative proportion of live-to-dead microbes. Data analysis was performed using the FACSDiva software v.5.0.2 (BD Biosciences).

\section{Collection and Treatment of Milk Samples}

Cow milk, both pre- (i.e., raw) and postpasteurization, were obtained from 6 industrial facilities around Ireland; 3 samples of each milk type were collected for analysis. Fresh unpasteurized milk was also obtained from the dairy herd at Teagasc Research Centre, Moorepark, and was pasteurized in-house using a Microthermics Heat exchanger (Microthermics, Wellington, CT) at $72^{\circ} \mathrm{C}$ for $15 \mathrm{~s}$ followed by rapid cooling to $4^{\circ} \mathrm{C}$. All milk samples were transported to the laboratory on ice before storage at $-20^{\circ} \mathrm{C}$. Milk samples were defrosted at $4^{\circ} \mathrm{C}$ before use.

\section{High-Throughput Sequencing and Bioinformatics Analysis}

Prior to extraction of DNA, milks (both raw and pasteurized) were treated with $100 \mu \mathrm{g} / \mathrm{mL}$ of nucleic acid stain ethidium monoazide (EMA; VWR, Dublin, Ireland; Rudi et al., 2005) to inactivate DNA not associated with living microbes (see supplementary material). Total DNA was then isolated from $1 \mathrm{~mL}$ of each raw and pasteurized milk sample using the PowerFood Microbial DNA extraction kit (MO BIO Laboratories, Carlsbad, CA) in accordance with manufacturer's instructions. Additionally, a 10-min incubation step at $70^{\circ} \mathrm{C}$ was incorporated to improve DNA yield, as described previously (Quigley et al., 2012a). The DNA extracts were used as a template for PCR amplification of $16 \mathrm{~S}$ rRNA tags (V4 region; 239 nucleotides long) using universal 16S rRNA-targeting primers predicted 
to bind to $94.6 \%$ of all $16 \mathrm{~S}$ rRNA genes, forward primer F1 (5'-AYTGGGYDTAAAGNG) and a combination of 4 reverse primers: R1 (5'-TACCRGGGTHTCTAATCC), R2 (5'-TACCAGAGTATCTAATTC), R3 (5'-CTACDSRGGTMTCTAATC), and R4 (5'-TACNVGGGTATCTAATC; RDP's Pyrosequencing Pipeline, http://pyro.cme.msu.edu/pyro/help.jsp). The PCR reaction contained $25 \mu \mathrm{L}$ of GoTaq Green Master Mix (Promega Corporation, Madison, WI), $1 \mu \mathrm{L}$ of each primer $(10 \mathrm{pmol}), 5 \mu \mathrm{L}$ of DNA template, and $\mathrm{H}_{2} \mathrm{O}$ to give a final reaction volume of $50 \mu \mathrm{L}$. The PCR amplification was performed using a G-Storm thermal cycler (Gene Technologies, Somerset, UK). Following a hot start of $10 \mathrm{~min}$ at $95^{\circ} \mathrm{C}$ to denature DNA, the amplification program consisted of an initial step at $94^{\circ} \mathrm{C}$ for $2 \mathrm{~min}$, followed by 40 cycles of denaturation at $94^{\circ} \mathrm{C}$ for $1 \mathrm{~min}$, annealing at $52^{\circ} \mathrm{C}$ for $1 \mathrm{~min}$, and extension at $72^{\circ} \mathrm{C}$ for $1 \mathrm{~min}$. A final elongation step at $72^{\circ} \mathrm{C}$ for $2 \mathrm{~min}$ was also included. The PCR amplicons were visualized on a $1.5 \%$ agarose gel. Amplicons were cleaned using the AMPure XP purification system (Beckman Coulter, Takeley, UK). The quantity of DNA extracted was assessed using the Quant-It Picogreen dsDNA reagent (Invitrogen) according to manufacturer's instructions and a Nanodrop 3300 Fluorospectrometer (Thermo Fisher Scientific Inc., Waltham, MA). The ND3300 excites in the presence of double-stranded DNA (dsDNA) bound with Picogreen at $470 \mathrm{~nm}$ and monitors emission at $525 \mathrm{~nm}$.

The 16S rRNA V4 amplicons were sequenced on the Teagasc454 Sequencing Platform (Genome Sequencer FLX; Roche Diagnostics Ltd., West Sussex, UK). Read processing was performed using techniques implemented in the RDP pyrosequencing pipeline (Cole et al., 2009). Sequences not passing the FLX quality controls were discarded, the 454 specific portion of the primer were trimmed, the raw sequences were sorted according to tag sequences, and reads with low quality scores (quality scores below 25) and short length (less than $150 \mathrm{bp}$ for the $16 \mathrm{~S}$ rRNA V4 region) were removed as well as reads that did not have exact matches with the primer sequence. Statistical analysis, to measure the sequence diversity included Chao1 richness, Shannon diversity, and rarefaction for monitoring sequencing abundance, were performed using the MOTHUR package (Schloss et al., 2009). Principal coordinate analysis (PCoA), measuring dissimilarities at phylogenetic distances based on Weighted Unifrac, was performed using the QIIME suite of programs (Caporaso et al., 2010). Trimmed fasta sequences were searched by BLAST (Altschul et al., 1990) against a previously published 16S-specific database (Urich et al., 2008) using default parameters. The resulting BLAST output was parsed using MEGAN (MEtaGenome ANalyzer; Huson et al.,
2007). MEGAN assigns reads to NCBI (National Center for Biotechnology Information) taxonomies by using the lowest common ancestor algorithm, which assigns each RNA-tag to the lowest common ancestor in the taxonomy from a subset of the best-scoring matches in the BLAST result. Bit scores were used from within MEGAN for filtering the results before tree construction and summarization (absolute cut-off: BLAST bitscore 86; relative cut-off: $10 \%$ of the top hit; Urich et al., 2008). Statistical significance was determined by the nonparametric Kruskal-Wallis test (Kruskal and Wallis, 1952) using Minitab (Coventry, UK) statistical package.

\section{Real-Time Quantitative PCR-Based Analysis}

To facilitate the absolute quantification of Pseudomonas in milk samples, a plasmid standard was first created. Here, a PCR product from within the $16 \mathrm{~S}$ rRNA gene of $P$. aeruginosa was generated using $16 \mathrm{~S}$ rRNA targeting, genus-specific primers (i.e., forward 5'-GACGGGTGAGTAATGCCTA-3' and reverse 5'-CACTGGTGTTCCTTCCTATA-3'; according to Spilker et al., 2004). Purified amplicons were cloned into the pCR2.1-TOPO vector using the TOPO-TA cloning system (Invitrogen) in accordance with manufacturer's instructions. Following cloning, the complete vector was transformed into chemically competent TOP-10 Escherichia coli cells (Invitrogen) and harvested on $\mathrm{LB}$ media containing $50 \mu \mathrm{g} / \mathrm{mL}$ of ampicillin. The accuracy of the cloned amplicon was confirmed by restriction analysis and DNA sequencing. The $\mathrm{qPCR}$ standards were prepared following the linearization of plasmid DNA with $K p n I$ restriction enzyme and quantification with the Nanodrop ND-1000 (Thermo Fisher Scientific Inc.). A standard curve was then generated via a series of dilutions from $10^{9}$ to $10^{2}$ copies $\mu \mathrm{L}^{-1}$ of DNA. The LightCycler 480 SYBR Green I Master kit (Roche Diagnostics GmbH, Mannheim, Germany) was used for quantification according to the manufacturer's instructions. Each PCR reaction contained $10 \mu \mathrm{L}$ of Sybr Green master mix, $1 \mu \mathrm{L}$ of both forward and reverse primer (10 pmol), $1 \mu \mathrm{L}$ of DNA, and was made up to a final volume of $20 \mu \mathrm{L}$ with nuclease-free $\mathrm{H}_{2} \mathrm{O}$. The PCR conditions were an initial denaturation at $95^{\circ} \mathrm{C}$ for $10 \mathrm{~min}$, followed by 35 cycles of denaturation at $95^{\circ} \mathrm{C}$ for $10 \mathrm{~s}$, annealing at $54^{\circ} \mathrm{C}$ for $5 \mathrm{~s}$, and elongation $72^{\circ} \mathrm{C}$ for $25 \mathrm{~s}$. The qPCR assays on test samples and controls were performed in triplicate. To facilitate quantification by qPCR it was necessary to first generate a standard curve relating colony-forming units to copy number of $16 \mathrm{~S}$ rRNA genes. Based on the amplification of DNA, which was isolated from Pseudomonas cultures of known cfu/mL $\left(3 \times 10^{7}, 3 \times 10^{6}\right.$, and $3 \times$ 
$10^{5} \mathrm{cfu} / \mathrm{mL}$ ), against a standard curve generated from the Lightcycler 480 (copy number $/ \mu \mathrm{L}$ ). A formula was generated to correct copy number values for $\mathrm{cfu} / \mathrm{mL}$ :

$$
\frac{(\mathrm{C} / \mu \mathrm{L})(\mathrm{TV})}{\mathrm{TCN}} \times \frac{\mathrm{T} \mathrm{cfu} / \mathrm{mL}}{1}=\mathrm{cfu} / \mathrm{mL}(\mathrm{S})
$$

where $\mathrm{C} / \mu \mathrm{L}=$ copy number $/ \mu \mathrm{L} ; \mathrm{TV}=$ template volume; $\mathrm{TCN}=$ total copy number of the standard used; $\mathrm{T}$ $\mathrm{cfu} / \mathrm{mL}=$ total $\mathrm{cfu} / \mathrm{mL}$ of standard used; and $\mathrm{cfu} / \mathrm{mL}$ $(\mathrm{S})=\mathrm{cfu} / \mathrm{mL}$ of the test sample. For example, results generated from comparing the copy number standard curve to DNA extracted from cultures of known cfu/ $\mathrm{mL}$ showed that an average value of, for example, 4.0 $\times 10^{9}$ amplicon copies corresponded to $3.2 \times 10^{8} \mathrm{cfu} /$ $\mathrm{mL}$ of $P$. aeruginosa; so, in the calculation above, TCN $=4.0 \times 10^{9}$ and $\mathrm{T} \mathrm{cfu} / \mathrm{mL}=3 \times 10^{8}$.

\section{RESULTS}

\section{Flow Cytometry Highlights the Proportion of Viable Microorganisms in Pasteurized Milk}

Flow cytometry was employed to compare the relative proportion of viable and dead cells in milk (both raw and pasteurized). Using this approach, the reduction in FITC fluorescence intensity when compared with control samples was deemed indicative of injury or death. The relative dot plot location of live and dead cells was first determined by spiking milk with what is regarded to be a thermosensitive species (i.e., L. lactis; Dumalisile et al., 2005; at $1 \times 10^{6} \mathrm{cfu} / \mathrm{mL}$; Figure 1A), as a control. As raw milk was used for these control studies, the innate microbiota of the milk remained intact. However, the high inoculum of Lactococcus ensured that the majority of events detected (>90\%) would correspond to L. lactis cells. Following the identification of viable cell populations (Figure 1A), positions occupied of injured or dead populations were determined following the extreme heat treatment of the L. lactis-spiked milk at $80^{\circ} \mathrm{C}$ for 30 min (Figure 1B). This extensive heat treatment resulted in the inactivation of the vast majority of cells present $(99.52 \%$ of events), with just a small number ( $0.48 \%$ of events) remaining in the live gated area. Having established the relative locations of viable and dead cells on this control plot, the focus switched to a direct comparison of raw and pasteurized milk. Raw milk was shown to contain mostly viable cells, but some dead or injured cells were also detected (Figure 1C). The cell populations present in the pasteurized milk sample were found to be predominantly nonviable; $10.7 \%$ were contained in the viable gate (Figure 1D). As expected, statistical analysis of these results revealed significant reductions in viable cell populations in pasteurized milk $(P<$ 0.01) compared with raw milk samples. Nonetheless, it is evident that a viable, microbial population was present in the pasteurized milk samples. To characterize this population, a high-throughput DNA sequencing strategy was initiated.

\section{High-Throughput Sequencing Reveals the Presence of Taxa Not Traditionally Regarded as Components of the Milk Microbiota}

Raw and pasteurized cow milk was obtained from 7 facilities located throughout Ireland. In each case the same milk was compared, both pre- and postpasteurization. To overcome the bias associated with the ability of PCR-based methods to amplify all DNA present, including DNA from dead cells, we incorporated the use of EMA. Following control tests of EMA (see supplementary material) we determined that the use of this stain is critical to ensure that DNA from dead cells does not lead to false positives. Following DNA extraction from these milk samples, amplicons of the $\mathrm{V} 4$ region of the $16 \mathrm{~S}$ rRNA gene were generated and sequence reads were obtained by high-throughput sequencing for phylogenetic assignment. A total of 48,837 pyrosequencing reads were obtained by 454 sequences. Diversity and richness estimations were calculated for each data set (Figure 2). The Chao1 estimator of species richness indicates good sample richness throughout. The Shannon diversity index, a measurement of overall diversity, indicates a diverse microbiota (Figure 2A). Rarefaction curve analysis revealed that the overall depth of sequencing is sufficient (Figure 2B). Kruskal-Wallis-based analysis of sequencing diversity values revealed that the values for raw and pasteurized milk samples are not significantly different. This highlights that, though pasteurization changed the number of bacteria present, the taxa present in the pasteurized milk are similar to those in the raw milk sample. This is also indicated by PCoA analysis, employing Weighted Unifrac distance matrix at operational taxonomic unit (OTU), which revealed no definitive split between raw and pasteurized milk samples (data not shown). When examined in combination, sequence reads corresponding to 4 distinct bacterial phyla (i.e., Proteobacteria, Bacteroidetes, Firmicutes, and Actinobacteria) were detected in raw milk, whereas 5 bacterial phyla (i.e., those present in raw milk in addition to Acidobacteria) were detected in pasteurized milk (Figures $3 \mathrm{~A}$ and $2 \mathrm{~B}$ ). Notably, reads corresponding to the fungal phylum Ascomycota were also detected at low levels in both raw and pasteurized milk. At the genus level, these fungal sequences corresponded almost exclusively to Penicillium 

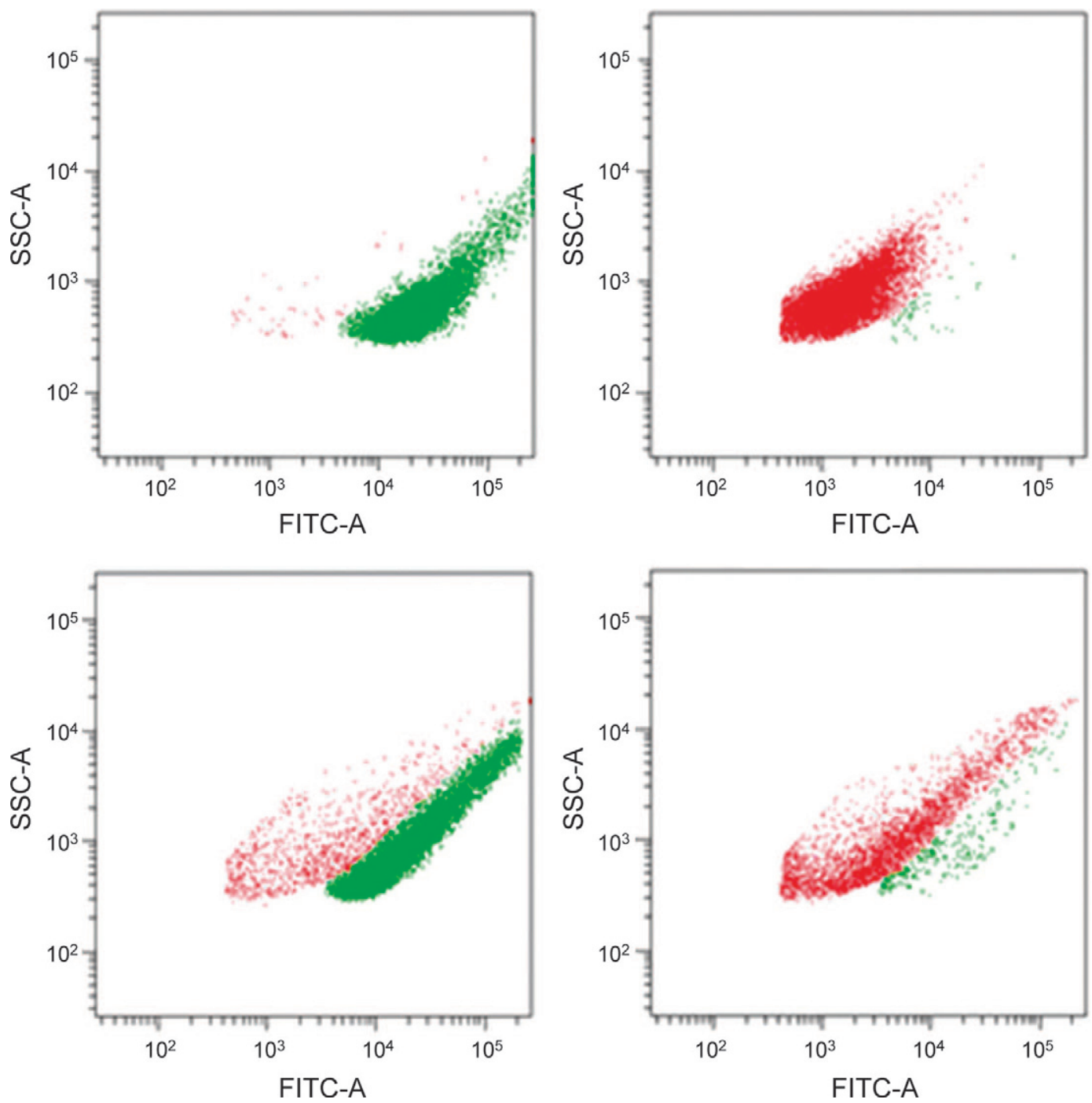

Figure 1. Flow cytometric analysis to determine the relative proportions of live and dead bacteria in milk. Treated milk spiked with $1 \times 10^{6}$ $\mathrm{cfu} / \mathrm{mL}$ Lactococcus lactis before (A; viable cell position) and after (B; dead cell position) heat treatment $\left(80^{\circ} \mathrm{C} 30 \mathrm{~min}\right)$. (C) Commercial raw milk containing mostly viable cells. (D) Commercially pasteurized milk samples in which a significant reduction in viable cells, compared with commercial raw milk, is apparent. In all cases the data are representative of triplicate data. SSC = side scatter; FITC = fluorescein isothiocyanate. Color version available in the online PDF.

spp. Further examination of the Penicillium sequences using BLAST established that they correspond to that of the mitochondrial 16S rRNA gene of Penicillium. Of the bacterial phyla, Firmicutes was found to dominate in both raw and pasteurized milk (80.51 and 84.04\%, respectively). A significantly greater proportion of Proteobacteria was observed in raw, relative to pasteurized, milk (9.20 vs. $5.75 \% ; P=0.02$ ), and a decrease in the proportions of Bacteroidetes and Actinobacteria were also apparent.

Further analysis of DNA sequence data (Figure 3C and 3D) highlighted that, at the family level, both raw and pasteurized milk were dominated by Streptococcaceae, with read abundance being $57 \%$ in raw milk and $77.69 \%$ in pasteurized milk. As expected, at the genus level these reads were found to predominantly correspond to Lactococcus spp. (Figure 3F; data corresponding to the individual milk facilities can be observed in supplementary material). A low proportion of Streptococcus spp. was also apparent. Other members of the lactic acid bacteria family (i.e., Leuconostocaceae and Lactobacillaceae) were also detected. The population most dramatically reduced as a consequence of pasteurization was Pseudomonaceae. These decreased significantly from $26.34 \%$ in raw milk to $4.1 \%$ in pasteurized milk $(P=0.055$; Figure $3 \mathrm{E}$ and $\mathrm{F})$. We further selected Pseudomonas for quantification by qPCR. The selection of Pseudomonas as a representative was as 
A

\begin{tabular}{lcc}
\hline Data Set & Raw Milk & Pasteurized Milk \\
\hline Similarity & $97 \%$ & $97 \%$ \\
$\begin{array}{l}\text { Chao1 richness } \\
\text { estimation }\end{array}$ & 1595 & 1977 \\
$\begin{array}{l}\text { Shannon index } \\
\text { for diversity }\end{array}$ & 6.41 & 6.59 \\
\hline
\end{tabular}

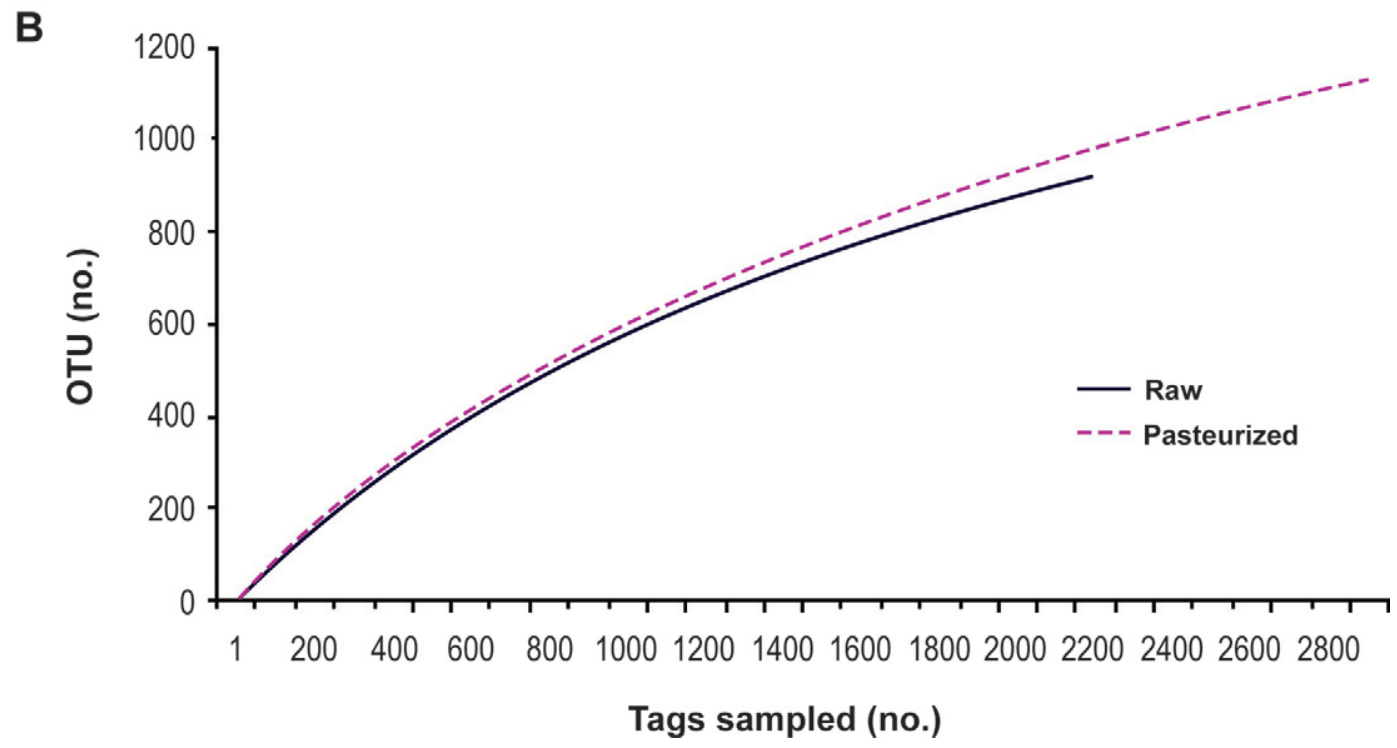

Figure 2. Analysis of the $\alpha$ diversity of the milk microbiota. (A) Rarefaction curve analysis of bacterial diversity. (B) Estimation of diversity of raw and pasteurized milk at the $97 \%$ similarity level; OTU = operational taxonomic unit. Color version available in the online PDF.

a consequence of its contribution to the spoilage of milk (Ternström et al., 1993) and the high-throughput sequencing-based data highlighting it as a species that decreased significantly in overall relative proportions following pasteurization. Raw milk was found to contain $1.21 \times 10^{4} \mathrm{cfu} / \mathrm{mL}$ of Pseudomonas on average, whereas the pasteurized samples showed a significant $(P<0.001)$ reduction in viable Pseudomonas, to $2.07 \times$ $10^{2} \mathrm{cfu} / \mathrm{mL}$. This reduction, though significant, is less dramatic than that observed following assessment of the efficacy of pasteurization on Pseudomonas numbers using culture based approaches, which demonstrated that pasteurization eliminates Pseudomonas from milk spiked with $1 \times 10^{6} \mathrm{cfu} / \mathrm{mL}$ of Pseudomonas aeruginosa (data not shown). Several other microbes often associated with dairy environments were also detected at low levels. These included the Brevibacteriaceae, Corynebacteriaceae, and Staphylococcaceae, all of which were identified in raw milk only. Several other families were found to be present in both raw and pasteurized milk. These included the Bifidobacteriaceae, Flavobacteriaceae, and Micrococcaceae, the latter corresponding to Arthrobacter at the genus level. Several typically gut-associated bacteria were also present in the milk samples. These included the Enterobacteriaceae, with reads corresponding to Cronobacter at the genus level. These were detected at low levels in pasteurized milk only, suggesting possible postpasteurization contamination. Also present were the Clostridiaceae and Ruminococcaceae, which, in the latter case, corresponded to the genera Ruminococcus and Faecalibacterium. Two families detected (i.e., Pasteurellaceae and Neisseriaceae), are more typically associated with the colonization of mucosal surfaces of many animals, whereas 2 families of more typically marine bacteria (i.e., Pseudoalteromonaceae and Vibrionaceae), were also detected. Finally, several other bacterial families not regarded as typical members of the milk microbiota were also detected. 
(a)
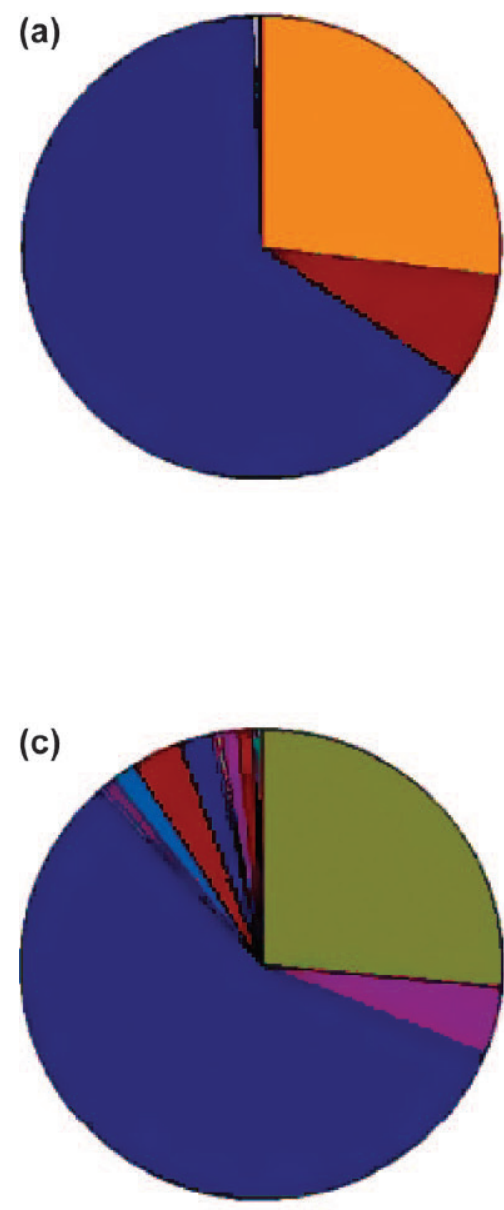

(e)

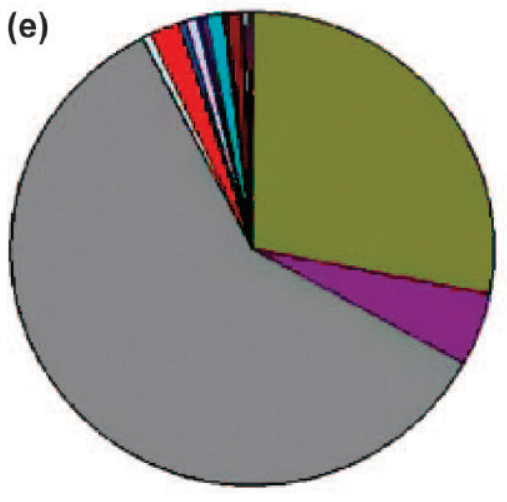

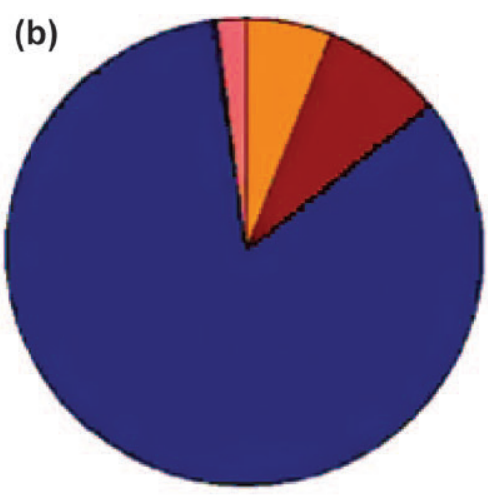

Proteobacteria

Bacteroidetes

Firmicutes

Ascomycota

Actinobacteria

- Acidobacteria

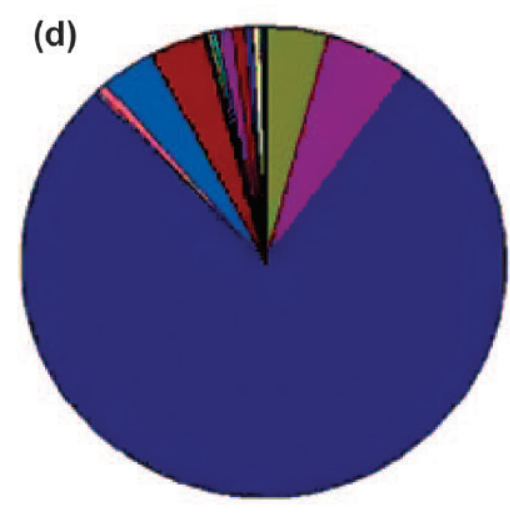

- Pseudomonadaceae

- Leuconostocaceae

- Streptococcaceae

$\square$ Trichocomaceae

- Pseudoalteromonadaceae

Enterobacteriaceae

- Prevotellaceae

- Bacteroidaceae

- Moraxellaceae

- Porphyromonadaceae

口 Veillonellaceae

Clostridiaceae

- Ruminococcaceae

- Flavobacteriaceae

- Micrococcaceae

Lactobacillaceae

- Bifidobacteriaceae

$\square$ Corynebacteriaceae

$\square$ Staphylococcaceae

$\square$ Pasteurellaceae

- Neisseriaceae

- Vibrionaceae

Pseudomonas

- Leuconostoc

- Lactococcus

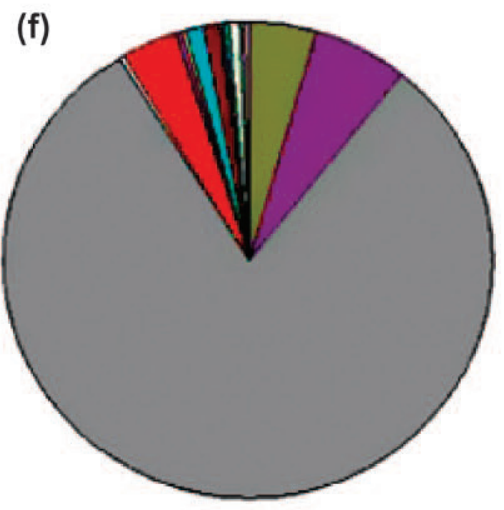

$\square$ Penicillium

- Pseudoalteromonas

Prevotella

- Psychrobacter

Bacteroides

- Parabacteroides

Acidaminococcus

$\square$ Clostridium

- Faecalibacterium

- Arthrobacter

- Flavobacterium

- Lactobaccifus

- Bifidobacterium

Ruminococcus

$\checkmark$ Staphylococcus

$\square$ Streptococcus

$\square$ Corynebacterium

$\checkmark$ Cronobacter

- Neisseria

- Vibrio

$\square$ Catenibacterium

Figure 3. Taxonomic assignment of the milk microbiota as determined by MEGAN (MEtaGenome ANalyzer). (a) Raw milk and phylum; (b) pasteurized milk and phylum; (c) raw milk and family; (d) pasteurized milk and family; (e) raw milk and genus; (f) pasteurized milk and genus. 
These include Bacteriodaceae, Porphyromonadaceae ( $P$ $=0.046)$, and Moraxcellaceae $(P=0.040)$. The latter corresponded to 2 genera (i.e., Acinetobacter and Psychrobacter), with Acinetobacter being found in raw milk only.

\section{DISCUSSION}

In this study we applied culture-independent techniques, including flow cytometry, high-throughput DNA sequencing, and qPCR, to uncover the bacterial profile of cow's milk both pre- and postpasteurization. Highthroughput DNA sequencing-based $16 \mathrm{~S}$ rRNA profiling revealed that cow's milk, both raw and pasteurized, contains a diverse microbial population. The microbial populations present in the milk samples differed according to the facility from which it was sourced (see supplementary material), but generally milk samples were dominated by lactic acid bacteria and Pseudomonas. Although many of the microbes detected are those previously associated with milk or dairy products, several of the bacteria isolated have not previously been detected in milk. These include Bacteroides, Parabacteroides, and Faecalibacterium, which are more frequently regarded as gut microbes that thrive in anaerobic environments, as well as Prevotella and Catenibacterium. The role of Bacteroides in gut health is controversial and has been the subject of much debate. It has been suggested that Bacteroides may play a significant role in the pathogenesis of allergies during infancy (Kirjavainen et al., 2001; Suzuki et al., 2008), whereas others have shown that it can provide protection against cow's milk allergies in mice trials (Rodriguez et al., 2012). The species Parabacteroides represents a reclassification of microbes that were previously regarded as being Bacteroides (Sakamoto and Benno, 2006). Faecalibacterium are commensal human gut bacteria with apparent anti-inflammatory activity (Sokol et al., 2008, 2009), which may ultimately be investigated with a view to its use as a probiotic to treat Crohn's disease, colitis, and irritable bowel syndrome (Kirjavainen et al. 2001). Prevotella are commensal microbes in rumen and hind gut of cows and sheep and in humans, where they help the breakdown of proteins and carbohydrates (Yildirim et al., 2010); it is also associated with periodontal disease in humans (Maeda et al., 1998). Although Catenibacterium are also associated with the human gut, little is known about this genus (Kageyama and Benno, 2000). The effect of these microbes on milk, milk products, or hosts, following the consumption of milk, remains unknown but, on the basis of these results, merits further attention.

Of the microbes previously associated with milk, psychrotolerant microorganisms present a considerable challenge to the dairy industry due to their ability to accelerate spoilage at storage conditions widely used in dairy production. Pseudomonas spp. are regarded as the predominant psychrotolerant bacteria in raw milk and a major contributor to milk spoilage. Previously, culture-based techniques suggested that these microbes are heat sensitive and usually do not survive pasteurization (Cousin, 1982; Ranieri et al., 2009). We confirmed that culture-based approaches indicate that pasteurization eliminates Pseudomonas from sterile milk spiked with $1 \times 10^{6} \mathrm{cfu} / \mathrm{mL}$ of $P$. aeruginosa. However, culture-independent methods reveal an overall reduction in, rather than elimination of, the Pseudomonas population following pasteurization, suggesting that damaged but nonviable cells are still present. Such cells are potentially metabolically active but are unable to be cultured on solid media. This finding is comparable to those revealed in a previous study (Gunasekera et al., 2002), which established that low numbers of Pseudomonas survive commercial pasteurization and may contribute to the Pseudomonas population in pasteurized milk. These and other pyschrotolerant bacteria may be overlooked when assessed by culture based approaches as a consequence of being in a viable but non culturable or highly stressed state.

\section{CONCLUSIONS}

In conclusion, this study reveals the existence of a diverse microbial population in cow milk, including the presence of several bacteria not previously observed in milk. Furthermore, changes in microbial diversity following pasteurization were seen to be less significant than previously expected following assessment of culture-based analysis. Finally, the data generated in this study suggests the potential for microbes, usually considered to be eliminated by pasteurization, to survive commercial pasteurization; this work suggests that these cells are in a highly stressed or viable but nonculturable state. Whereas the reductions in numbers which does occur, combined with the stressed state of surviving cells, ensures the safety of pasteurized milk, the apparent presence of these populations in commercial milk, and their potential effect on milk quality, shelf-life, and milk-based products is intriguing and merits closer attention.

\section{ACKNOWLEDGMENTS}

Lisa Quigley and Robert McCarthy were supported by Teagasc Walsh Fellowships. The authors thank Fiona Crispie (Teagasc Food Research Centre, Moorepark), who carried out high-throughput sequencing analysis, and Vincenzo Fallico (Teagasc Food Research 
Centre, Moorepark) for his help with flow cytometry. This study was funded by FP7 EFRAIM FP7-KBBE2007-1-'Mechanisms of early protective exposures on allergy development' and in part by Department of Agriculture, Fisheries and Food (Dublin, Ireland) under the National Development Plan 2007-2013.

\section{REFERENCES}

Alegría, A., P. Szczesny, B. Mayo, J. Bardowski, and M. Kowalczyk. 2012. Biodiversity in Oscypek, a traditional Polish cheese, determined by culture-dependent and -independent approaches. Appl. Environ. Microbiol. 78:1890-1898.

Altschul, S. F., W. Gish, W. Miller, E. W. Myers, and D. J. Lipman. 1990. Basic local alignment search tool. J. Mol. Biol. 215:403-410.

Andersson, A. F., M. Lindberg, H. Jakobsson, F. Backhed, P. Nyren, and L. Engstrand. 2008. Comparative analysis of human gut microbiota by barcoded pyrosequencing. PLoS ONE 3:e2836.

Braem, G., S. De Vliegher, B. Verbist, M. Heyndrickx, F. Leroy, and L. De Vuyst. 2012. Culture-independent exploration of the teat apex microbiota of dairy cows reveals a wide bacterial species diversity. Vet. Microbiol. 157:383-390.

Callon, C., F. Duthoit, C. Delbes, M. Ferrand, Y. Le Frileux, R. De Cremoux, and M. C. Montel. 2007. Stability of microbial communities in goat milk during a lactation year: Molecular approaches. Syst. Appl. Microbiol. 30:547-560.

Caporaso, J. G., J. Kuczynski, J. Stombaugh, K. Bittinger, F. D. Bushman, E. K. Costello, N. Fierer, A. G. Pena, J. K. Goodrich, J. I. Gordon, G. A. Huttley, S. T. Kelley, D. Knights, J. E. Koenig, R. E. Ley, C. A. Lozupone, D. McDonald, B. D. Muegge, M. Pirrung, J. Reeder, J. R. Sevinsky, P. J. Tumbaugh, W. A. Walters, J. Widmann, T. Yatsunenko, J. Zaneveld, and R. Knight. 2010 QIIME allows analysis of high-throughput community sequencing data. Nat. Methods 7:335-336.

Cole, J. R., Q. Wang, E. Cardenas, J. Fish, B. Chai, R. J. Farris, A. S. Kulam-Syed-Mohideen, D. M. McGarrell, T. Marsh, G. M. Garrity, and J. M. Tiedje. 2009. The ribosomal database project: Improved alignments and new tools for rRNA analysis. Nucleic Acids Res. 37:D141-D145.

Cousin, M. A. 1982. Presence and activity of psychrotrophic microorganisms in milk and dairy products: A review. J. Food Prot. 45:172-207.

Delmont, T. O., E. Prestat, K. P. Keegan, M. Faubladier, P. Robe, I. M. Clark, E. Pelletier, P. R. Hirsch, F. Meyer, J. A. Gilbert, D. Le Paslier, P. Simonet, and T. M. Vogel. 2012. Structure, fluctuation and magnitude of a natural grassland soil metagenome. ISME J. 6:1677-1687.

Dobson, A., O. O'Sullivan, P. D. Cotter, P. Ross, and C. Hill. 2011. High-throughput sequence-based analysis of the bacterial composition of kefir and an associated kefir grain. FEMS Microbiol. Lett. 320:56-62

Dumalisile, P., R. C. Witthuhn, and T. J. Britz. 2005. Impact of different pasteurization temperatures on the survival of microbial contaminants isolated from pasteurized milk. Int. J. Dairy Technol. $58: 74-82$

Fromm. H. I., and K. J. Boor. 2004. Characterization of pasteurized fluid milk shelf-life attributes. J. Food Sci. 69:M207-M214.

Gunasekera, T. S., P. V. Attfield, and D. A. Veal. 2000. A flow cytometry method for rapid detection and enumeration of total bacteria in milk. Appl. Environ. Microbiol. 66:1228-1232.

Gunasekera, T. S., A. Sorensen, P. V. Attfield, S. J. Sorensen, and D. A. Veal. 2002. Inducible gene expression by nonculturable bacteria in milk after pasteurization. Appl. Environ. Microbiol. 68:19881993.

He, H., J. Dong, C. N. Lee, and Y. Li. 2009. Molecular analysis of spoilage-related bacteria in pasteurized milk during refrigeration by PCR and denaturing gradient gel electrophoresis. J. Food Prot. $72: 572-577$.
Huson, D. H., A. F. Auch, J. Qi, and S. C. Schuster. 2007. MEGAN analysis of metagenomic data. Genome Res. 17:377-386.

Kageyama, A., and Y. Benno. 2000. Catenibacterium mitsuokai gen. nov., sp. nov., a gram-positive anaerobic bacterium isolated from human faeces. Int. J. Syst. Evol. Microbiol. 50:1595-1599.

Kirjavainen, P. V., E. Apostolou, T. Arvola, S. J. Salminen, G. R. Gibson, and E. Isolauri. 2001. Characterizing the composition of intestinal microflora as a prospective treatment target in infant allergic disease. FEMS Immunol. Med. Microbiol. 32:1-7.

Kruskal, W. H., and A. W. Wallis. 1952. Use of ranks in one-criterion variance analysis. J. Am. Stat. Assoc. 47:583-621.

Maeda, N., M. Okamoto, K. Kondo, H. Ishikawa, R. Osada, A. Tsurumoto, and H. Fujita. 1998. Incidence of Prevotella intermedia and Prevotella nigrescens in periodontal health and disease. Microbiol. Immunol. 42:583-589.

Masoud, W., M. Takamiya, F. K. Vogensen, S. Lillevang, W. Abu Al-Soud, S. J. Sorensen, and M. Jakobsen. 2011. Characterization of bacterial populations in Danish raw milk cheeses made with different starter cultures by denaturating gradient gel electrophoresis and pyrosequencing. Int. Dairy J. 21:142-148.

Paszyn'ska-Wesołowska, I., and M. Bartoszcze. 2009. Bacteria in the state of VBNC-A threat to human health. Med. Weter. 65:228 231.

Quigley, L., O. O'Sullivan, T. P. Beresford, R. Paul Ross, G. F. Fitzgerald, and P. D. Cotter. 2012a. A comparison of methods used to extract bacterial DNA from raw milk and raw milk cheese. J. Appl. Microbiol. 113:96-105.

Quigley, L., O. O'Sullivan, T. P. Beresford, R. Paul Ross, G. F. Fitzgerald, and P. D. Cotter. 2012b. High-throughput sequencing detects subpopulations of bacteria not previously associated with artisanal cheeses. Appl. Environ. Microbiol. 78:5717-5723.

Quigley, L., O. O'Sullivan, T. P. Beresford, R. P. Ross, G. F. Fitzgerald, and P. D. Cotter. 2011. Molecular approaches to analysing the microbial composition of raw milk and raw milk cheese. Int. J. Food Microbiol. 150:81-94.

Ranieri, M. L., J. R. Huck, M. Sonnen, D. M. Barbano, and K. J. Boor. 2009. High temperature, short time pasteurization temperatures inversely affect bacterial numbers during refrigerated storage of pasteurized fluid milk. J. Dairy Sci. 92:4823-4832.

Rodriguez, B., G. Prioult, F. Hacini-Rachinel, D. Moine, A. Bruttin, C. Ngom-Bru, C. Labellie, I. Nicolis, B. Berger, A. Mercenier, M. J. Butel, and A. J. Waligora-Dupriet. 2012. Infant gut microbiota is protective against cow's milk allergy in mice despite immature ileal T-cell response. FEMS Microbiol. Ecol. 79:192-202.

Rodríguez-Lázaro, D., M. D'Agostino, A. Herrewegh, M. Pla, N. Cook, and J. Ikonomopoulos. 2005. Real-time PCR-based methods for detection of Mycobacterium avium subsp. paratuberculosis in water and milk. Int. J. Food Microbiol. 101:93-104.

Rudi, K., B. Moen, S. M. Drømtorp, and A. L. Holck. 2005. Use of ethidium monoazide and PCR in combination for quantification of viable and dead cells in complex samples. Appl. Environ. Microbiol. 71:1018-1024.

Sakamoto, M., and Y. Benno. 2006. Reclassification of Bacteroides distasonis, Bacteroides goldsteinii and Bacteroides merdae as Parabacteroides distasonis gen. nov., comb. nov., Parabacteroides goldsteinii comb. nov and Parabacteroides merdae comb. nov. Int. J. Syst. Evol. Microbiol. 56:1599-1605.

Schloss, P. D., S. L. Westcott, T. Ryabin, J. R. Hall, M. Hartmann E. B. Hollister, R. A. Lesniewski, B. B. Oakley, D. H. Parks, C. J. Robinson, J. W. Sahl, B. Stres, G. G. Thallinger, D. J. Van Horn, and C. F. Weber. 2009. Introducing mothur: Open-source, platform-independent, community-supported software for describing and comparing microbial communities. Appl. Environ. Microbiol. 75:7537-7541.

Sogin, M. L., H. G. Morrison, J. A. Huber, D. Mark Welch, S. M Huse, P. R. Neal, J. M. Arrieta, and G. J. Herndl. 2006. Microbial diversity in the deep sea and the underexplored "rare biosphere". Proc. Natl. Acad. Sci. USA 103:12115-12120.

Sokol, H., B. Pigneur, L. Watterlot, O. Lakhdari, L. G. BermudezHumaran, J. J. Gratadoux, S. Blugeon, C. Bridonneau, J. P. Furet, G. Corthier, C. Grangette, N. Vasquez, P. Pochart, G. Trugnan, 
G. Thomas, H. M. Blottiere, J. Dore, P. Marteau, P. Seksik, and P. Langella. 2008. Faecalibacterium prausnitzii is an anti-inflammatory commensal bacterium identified by gut microbiota analysis of Crohn disease patients. Proc. Natl. Acad. Sci. USA 105:1673116736.

Sokol, H., P. Seksik, J. P. Furet, O. Firmesse, L. Nion-Larmurier, L. Beaugerie, J. Cosnes, G. Corthier, P. Marteau, and J. Dore. 2009. Low counts of Faecalibacterium prausnitzii in colitis microbiota. Inflamm. Bowel Dis. 15:1183-1189.

Spilker, T., T. Coenye, P. Vandamme, and J. J. LiPuma. 2004. PCRbased assay for differentiation of Pseudomonas aeruginosa from other Pseudomonas species recovered from cystic fibrosis patients. J. Clin. Microbiol. 42:2074-2079.

Suzuki, S., N. Shimojo, Y. Tajiri, M. Kurnernura, and Y. Kohno. 2008. A quantitative and relative increase in intestinal bacteroides in allergic infants in rural Japan. Asian Pac. J. Allergy Immunol. 26:113-119.

Ternström, A., A. M. Lindberg, and G. Molin. 1993. Classification of the spoilage flora of raw and pasteurized bovine-milk, with special reference to Pseudomonas and Bacillus. J. Appl. Bacteriol. $75: 25-34$.
Urich, T., A. Lanzen, J. Qi, D. H. Huson, C. Schleper, and S. C. Schuster. 2008. Simultaneous assessment of soil microbial community structure and function through analysis of the meta-transcriptome. PLoS ONE 3:e2527.

Vacheyrou, M., A. C. Normand, P. Guyot, C. Cassagne, R. Piarroux, and Y. Bouton. 2011. Cultivable microbial communities in raw cow milk and potential transfers from stables of sixteen French farms. Int. J. Food Microbiol. 146:253-262.

Verdier-Metz, I., G. Gagne, S. Bornes, F. Monsallier, P. Veisseire, C. Delbès-Paus, and M. C. Montel. 2012. Cow teat skin, a potential source of diverse microbial populations for cheese production. Appl. Environ. Microbiol. 78:326-333.

Verdier-Metz, I., V. Michel, C. Delbès, and M. C. Montel. 2009. Do milking practices influence the bacterial diversity of raw milk? Food Microbiol. 26:305-310.

Yildirim, S., C. J. Yeoman, M. Sipos, M. Torralba, B. A. Wilson, T. L. Goldberg, R. M. Stumpf, S. R. Leigh, B. A. White, and K. E. Nelson. 2010. Characterization of the fecal microbiome from nonhuman wild primates reveals species specific microbial communities. PLoS ONE 5:e13963. 